\title{
High-Rayleigh-Number Convection in a Vertical Channel
}

\author{
M. Gibert, ${ }^{*}$ H. Pabiou, F. Chillà, and B. Castaing \\ Laboratoire de Physique, CNRS UMR 5672, Ecole Normale Supérieure de Lyon, 46 Allée d'Italie, 69364 Lyon Cedex 7 , France
}

(Received 22 August 2005; published 28 February 2006)

\begin{abstract}
We measure the relation between convective heat flux and temperature gradient in a vertical channel filled with water, the average vertical mass flux being zero. Compared to the classical Rayleigh-Bénard case, this situation has the advantage of avoiding plates and, thus, their neighborhood, in which is usually concentrated most of the temperature gradient. Consequently, inertial processes should control the convection, with poor influence of the viscosity. This idea gives a good account of our observations, if we consider that a natural vertical length, different from the channel width, appears. Our results also suggest that heat fluxes can be deduced from velocity measurements in free convective flows. This confers to our results a wide range of applications.
\end{abstract}

DOI: 10.1103/PhysRevLett.96.084501

PACS numbers: 47.27.T-, 95.30.Tg

Convective flows have been studied for a long time [13]. The most studied laboratory situation is the RayleighBénard (RB) convection, in which a fluid is confined between two horizontal plates. However, in this case, the heat transfer is controlled mainly by the neighborhood of the plates, where most of the temperature gradient is concentrated. In this respect, it does not correspond well to many interesting situations such as convection in stars or planets, where such a confinement does not exist.

We present here the first experimental study of a new paradigm, which should better correspond to these situations: heat flow in a vertical, infinitely long channel with no vertical mass transfer. Obviously, in experimental realizations, the channel is not infinite. It does correspond well to a quarrel pit as far as it is the only access to underground galleries, to prevent large scale circulation. Indeed, one of the works which has triggered our curiosity is the study of cold avalanches in a quarrel pit by Perrier et al. [4]. The recent numerical study of Lohse and Toschi [3,5] also inspired our experiment. Other related works are Refs. [6-8], which study the buoyant mixing of miscible fluids in a vertical tube, with unstable density gradient, but there the average vertical mass flux is not zero.

When compared to the RB case, the ideal case of the infinite channel, with its assumed translational invariance along the vertical direction, also asks for slightly different definitions of the Nusselt $(\mathrm{Nu})$ and the Rayleigh ( $\mathrm{Ra}$ ) numbers. $\beta$ being the average vertical temperature gradient:

$$
\mathrm{Nu}=\frac{Q}{\lambda \beta},
$$

where $\lambda$ is the thermal conductivity of the fluid, and $Q$ is the heat flux. Thus, as usual, Nu compares the heat flux to its value in a purely conductive situation.

The Rayleigh number can be chosen as:

$$
\mathrm{Ra}=\frac{g \alpha \beta d^{4}}{\nu \kappa},
$$

where $\alpha$ is the isobaric thermal expansion coefficient, $\nu$ is the kinematic viscosity, $\kappa$ is the heat diffusivity, and $g$ is the gravity acceleration. $d$ is a characteristic length, which could be the transverse size of the channel.

Our experimental setup, represented in Fig. 1, is constituted mainly of a traditional rectangular RayleighBénard cell of height $40 \mathrm{~cm}$ and section $40 \times 10 \mathrm{~cm}^{2}$. Thanks to a honeycomb structure (made in polyvinylchloride), we avoid convection appearing in about $50 \%$ of the RB cell. The other 50\%, here called the zone of convection, has in its central part a channel with a $10 \times 10 \mathrm{~cm}^{2}$ cross section area and a height of $20 \mathrm{~cm}$. This channel will be our zone of interest. The fluid is water and the walls of the RB cell are $25 \mathrm{~mm}$ thick polymethylmethacrylate. As usual, the upper plate of the cell is temperature regulated by a

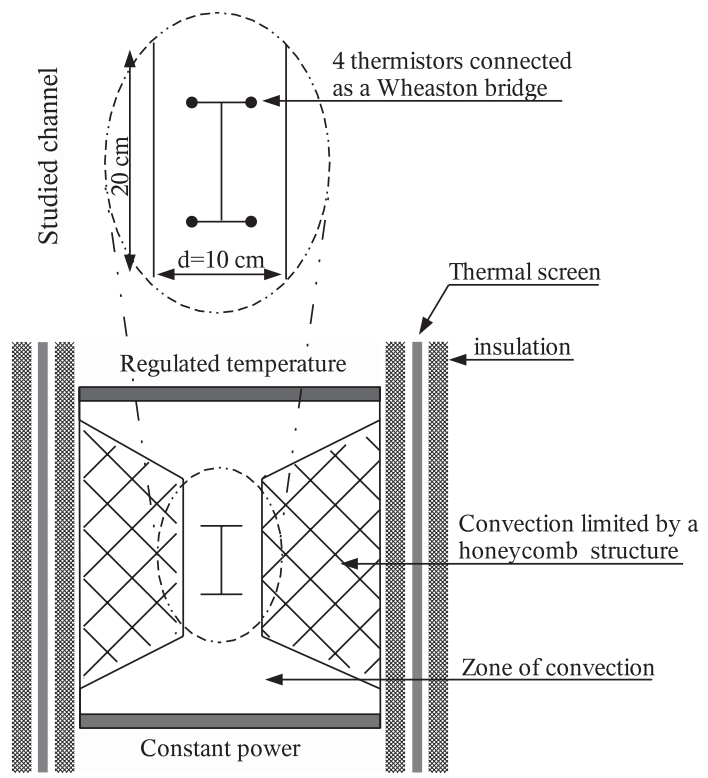

FIG. 1. Schematic representation of the experimental setup. The circles in the studied channel represent the resistors of the bridge. 
regulated water bath. The bottom plate is heated by Joule effect in 5 resistors, regularly spaced. The total power input could go from 10 to $300 \mathrm{~W}$ to cover our range of Rayleigh numbers. The cell is thermally insulated and surrounded by a copper screen. This screen is temperature regulated at the average temperature between the top and bottom plates, which is maintained constant on a whole set of measurements. As a result of the precise procedure described below, we consider that heat losses, and thus the useful power, are known within $\pm 0.5 \mathrm{~W}$.

Our main setup for measurements (the bridge) uses four resistive thermometers from the same batch [9] located at the corners of a rectangle of $10 \mathrm{~cm}$ high and $5 \mathrm{~cm}$ wide. To maintain this geometry without being too intrusive, we use an "I" structure as shown in Fig. 1 zoom. The resistors are connected as a Wheaston bridge, in such a way that the two upper (lower) ones are in the opposite situation (no common point). As the temperature dependencies of the resistors are very close, the output of the bridge is poorly sensitive to the average temperature but very sensitive to a temperature difference between the top and bottom branches of the bridge. The input of the bridge is $30 \mathrm{~Hz}$, $0.1 \mathrm{~V}$ voltage from an Agilent 33220A generator. The output is measured by a differential lock-in amplifier (Stanford SR830 DSP). The sensitivity of the bridge to temperature differences is typically $2 \mathrm{mV} / \mathrm{K}$, and the order of magnitude of the output for zero temperature difference (the bridge offset) is $10^{-4} \mathrm{~V}$.

Heat losses have been estimated in situ, looking at the root mean squared amplitude of fluctuations of the bridge output and extrapolating the supplied power for zero fluctuations. Such an extrapolation also gave the exact output of the bridge for a zero temperature gradient in the channel.

As presented in the introduction, we consider our $20 \mathrm{~cm}$ long channel as a good approximation of an infinite channel. We also consider that our temperature difference measured in the center of the channel is a good approximation of the temperature difference produced, along $10 \mathrm{~cm}$, by the gradient occurring in this infinite channel, with the same heat flux. In order to check these hypotheses, we used a shorter bridge $5 \mathrm{~cm}$ high. With this bridge, we measured the temperature gradient all along the vertical axis of the cell, the average temperature being $30^{\circ} \mathrm{C}$, and the power $200 \mathrm{~W}$. Each measurement is averaged over 24 hours with $1 \mathrm{~Hz}$ acquisition frequency. The data, presented in Fig. 2, show that a relatively strong jump in temperature occurs close to both ends of the channel. Therefore, we model the temperature profile by a constant slope in the center and with two jumps on the edges of the channel. To take into account the length on which each edge has an influence, we transform this ideal profile through a first order filter [10] (see inset in Fig. 2). With this filtered profile, we calculate the temperature difference on a $5 \mathrm{~cm}$ distance. We choose the jump, the slope, and the characteristic length of the filter to fit our data. Also shown in Fig. 2 are the assumed

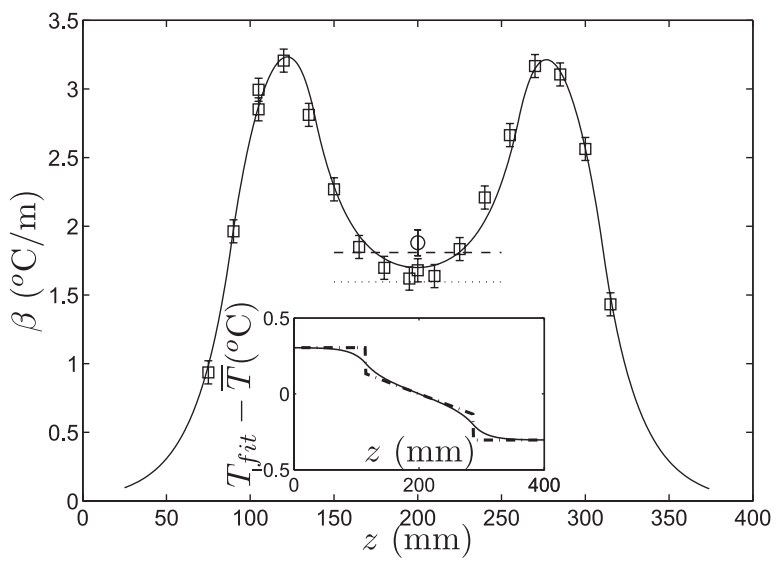

FIG. 2. Vertical gradient versus the height on the vertical axis of the cell. The heat power is $200 \mathrm{~W}$. Squares: Measurements with the $5 \mathrm{~cm}$ bridge. Circle: Measurement with the $10 \mathrm{~cm}$ bridge. Continuous line: Fit with the model temperature profile (see the continuous line in the inset). Dotted line: Corresponding to the infinite channel gradient in the center of the cell. Dashed line: Corresponding to the $10 \mathrm{~cm}$ temperature difference in the center of the cell. The inset exhibits the temperature profile along the $z$ axis ( $\bar{T}$ being the mean temperature). Dashed-dotted line: Ideal profile which corresponds in the central part to the infinite channel. Continuous line: Filtered profile, used to fit measurements, to take into account the finite length of the channel.

infinite channel gradient (dotted horizontal line), the $10 \mathrm{~cm}$ distance temperature difference, centered on the middle of the channel (dashed horizontal line), and the result of the measurement with the $10 \mathrm{~cm}$ bridge (circle). This fit suggests that our measurements with the $10 \mathrm{~cm}$ bridge give gradient (Nusselt) values approximately 10\% larger (smaller) than for the infinite channel. However, this $10 \mathrm{~cm}$ bridge is the best compromise between this finite length effect and the measurement accuracy we can reach. Indeed, with this kind of bridge, we can measure a temperature difference within $\pm 5 \times 10^{-3}{ }^{\circ} \mathrm{C}$.

Now we come back to the main measurements, with the $10 \mathrm{~cm}$ cross in the middle of the channel. Three different global informations can be obtained from recording the output of the bridge (acquisition frequency: $1 \mathrm{~Hz}$ ). The average on 24 hours recording gives the average gradient in the channel. The root mean squared amplitude of fluctuations gives a characteristic temperature amplitude $\theta$. Finally, the power spectrum of the fluctuations shows a characteristic cutoff frequency $f_{c}$ or a characteristic time $\tau_{c}=1 / 2 \pi f_{c}$. This time is interpreted as a large scale correlation time, giving information on the velocity of thermal structures.

The measured temperature gradient $\beta$ gives us the Nusselt number, Eq. (1), where $Q$ is now the power supplied $(P)$ minus the heat losses $\left(P_{\text {losses }}\right)$, divided by the cross section area of the channel $\left(S=10^{-2} \mathrm{~m}^{2}\right): Q=$ $\left(P-P_{\text {losses }}\right) / S$. Three sets of measurements were made, 
at three different average temperatures, 20,30 , and $40{ }^{\circ} \mathrm{C}$. They correspond to three different Prandtl numbers: $\operatorname{Pr}\left(20^{\circ} \mathrm{C}\right)=7, \operatorname{Pr}\left(30^{\circ} \mathrm{C}\right)=5.3$, and $\operatorname{Pr}\left(40^{\circ} \mathrm{C}\right)=4$. The Prandtl number $\operatorname{Pr}=\nu / \kappa$ is the ratio of the kinematic viscosity $\nu$ to the heat diffusivity $\kappa$.

To define the Reynolds number Re and the Rayleigh number Ra, we need a characteristic length. We first used the width of the channel, $d=10 \mathrm{~cm}$, defining:

$$
\operatorname{Re}=\frac{d^{2}}{\nu \tau_{c}} ; \quad \operatorname{Ra}=\frac{g \alpha \beta d^{4}}{\nu \kappa}
$$

Figure 3 shows both $\mathrm{Nu}$ and $\mathrm{RePr}$ versus RaPr. While the three sets of values correctly merge for RePr, they do not for $\mathrm{Nu}$. This is particularly clear for the highest Ra values, for which experimental uncertainties are negligible. Hence, while the RePr versus RaPr convergence seems to indicate that the kinematic viscosity has no influence (pure inertial behavior), the $\mathrm{Nu}$ results seem to push the opposite way.

However, there is no fundamental reason imposing $d$ as the correlation length. Another possible choice is using the amplitude $\theta$ of temperature fluctuations. Indeed, $\theta$, which is the root mean square of the bridge output, has the meaning of a temperature only if the correlation length of the temperature fluctuations is smaller than the height of the bridge $(10 \mathrm{~cm})$. Otherwise, $\theta$ would represent a gradient rms.

Assuming this short correlation length hypothesis, we can consider $\theta / \beta$ as a natural characteristic length, which should be proportional to the correlation length $L: L=$ $K \theta / \beta$. To determine $K$, we compare the typical velocity $v=L / \tau_{c}=K \theta / \beta \tau_{c}$ with a particle imaging velocimetry [11] analysis of the flow, without the thermal screen. For this last measure, we worked with the mean temperature of the fluid being equal to the room temperature $\left(30^{\circ} \mathrm{C}\right)$ and a relatively high heat power $(200 \mathrm{~W})$, which is quite bigger

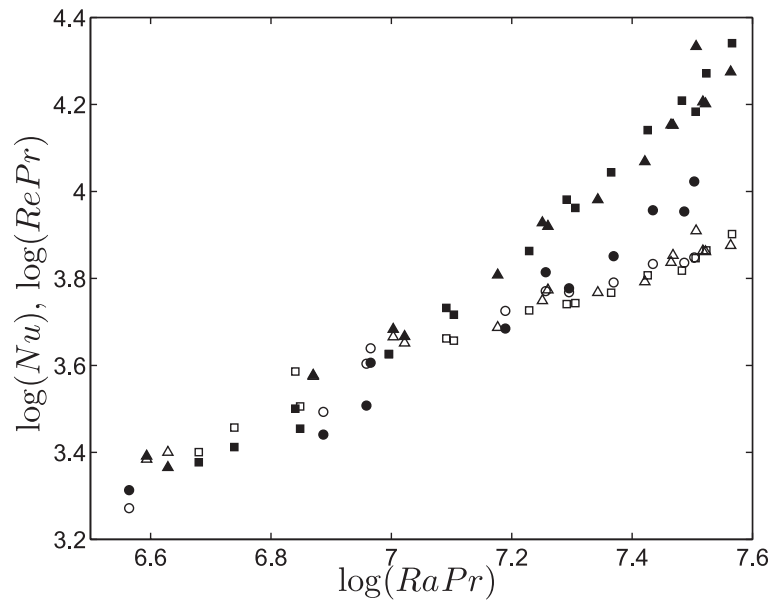

FIG. 3. Solid symbols: Nusselt number. Open symbols: Reynolds number. Circles: $20^{\circ} \mathrm{C}(\mathrm{Pr}=7)$; triangles: $30^{\circ} \mathrm{C}(\mathrm{Pr}=$ 5.3); squares: $40^{\circ} \mathrm{C}(\mathrm{Pr}=4)$. than heat losses. Identifying $v$ with the observed rms velocity $(0.5 \mathrm{~cm} / \mathrm{s})$ gives $K \simeq 1 / 2$ and $L=\theta / 2 \beta$. It is worth noting that Debacq et al. [7] introduce the same characteristic length. However, their very different conditions in terms of Prandtl (or Schmidt) and Reynolds numbers result in behaviors hardly comparable with ours.

Using $L$ as characteristic length renormalizes the Reynolds and Rayleigh numbers, which take the "natural" values $\mathrm{Re}_{n}$ and $\mathrm{Ra}_{n}$ :

$$
\begin{gathered}
\operatorname{Re}_{n}=\frac{L^{2}}{\nu \tau_{c}}=\operatorname{Re}\left(\frac{\theta}{2 \beta d}\right)^{2} ; \\
\operatorname{Ra}_{n}=\frac{g \alpha \beta L^{4}}{\nu \kappa}=\operatorname{Ra}\left(\frac{\theta}{2 \beta d}\right)^{4} .
\end{gathered}
$$

Using these natural values improves a lot the merging of the three sets of data in a $\operatorname{Re}_{n} \operatorname{Pr}$ versus $\mathrm{Ra}_{n} \operatorname{Pr}$ plot, as shown in Fig. 4. Moreover, in the same Fig. 4, these three sets reasonably merge for $\mathrm{Nu}$ versus $\mathrm{Ra}_{n} \mathrm{Pr}$, confirming the inertial character of the convection.

The slope of the loglog plot of $\operatorname{Re}_{n} \operatorname{Pr}$ versus $\operatorname{Ra}_{n} \operatorname{Pr}$ is very close to 0.5 , in agreement with both expectations and the Lohse and Toschi results. The corresponding slope for $\mathrm{Nu}$ is closer to 0.6. However, the difference with 0.5 cannot be certified. We present on the graph error bars due to a $0.5 \mathrm{~W}$ uncertainty on the heat losses.

This merging of data suggests that all the influence of the viscosity (and, thus, of the Reynolds number) is hidden in the behavior of the length $L$ that we have introduced. Furthermore, it is interesting to look at the dependence of this length with the Reynolds number behavior shown in Fig. 5. Once more, all data seem to merge on a single curve. It shows a kind of crossover for a Reynolds number of order of magnitude 250. For $\operatorname{Re}_{n}<250$, the length $L$ is constant, close to half the width of the channel. For

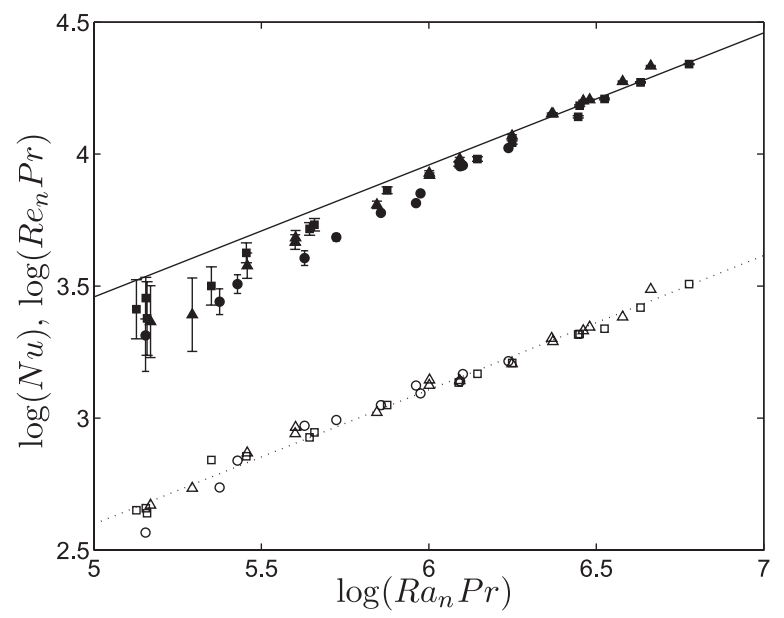

FIG. 4. With $\operatorname{Re}_{n}$ and $\mathrm{Ra}_{n}$ based on the coherence length $L=$ $\theta / 2 \beta$, the experiments at different Prandtl merge. Same symbols as in Fig. 3. The dotted line corresponds to $\operatorname{Re}_{n} \operatorname{Pr}=$ $1.14 \sqrt{\mathrm{Ra}_{n} \operatorname{Pr}}$ and the solid one to $\mathrm{Nu}=9.1 \sqrt{\mathrm{Ra}_{n} \operatorname{Pr}}$. 


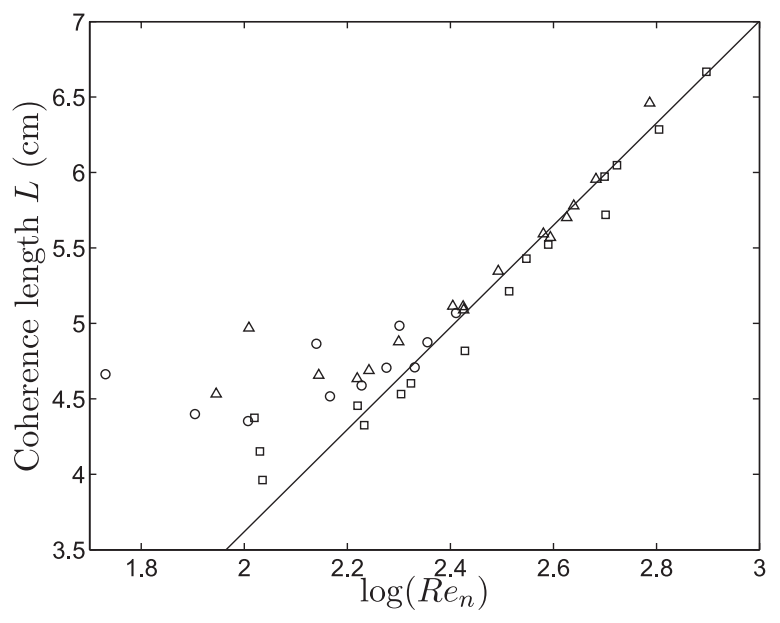

FIG. 5. Behavior of the coherence length $L$ versus $\ln \mathrm{Re}_{n}$. Same symbols as in Fig. 3. The line corresponds to $L / d=$ $0.14 \ln \left(\operatorname{Re}_{n}\right)-0.3$.

$\operatorname{Re}_{n}>250$, the length $L$ seems to take a linear behavior with $\ln \operatorname{Re}_{n}$.

Put in another way, the two relations $\mathrm{Nu} \propto\left(\mathrm{Ra}_{n} \operatorname{Pr}\right)^{1 / 2}$ and $\operatorname{Re} \operatorname{Pr} \propto\left(\operatorname{Ra}_{n} \operatorname{Pr}\right)^{1 / 2}$, together with the above linear dependence of $L / d$ in $\operatorname{lnRe}_{n}$, are equivalent to:

$$
Q \propto C v \theta \text { and } v \propto \sqrt{g \alpha \beta d^{2}} \ln \operatorname{Re}_{n},
$$

where $C$ is the specific heat per unit volume, and $v$ is the typical velocity of the fluid. The first relation is difficult to avoid. The logarithmic correction in the second one could be related to the existence of a logarithmic boundary layer along the walls, which is not present in the numerical experiment of Lohse and Toschi [5], but the verification of this assumption is out of the scope of this Letter. The additional output of our experiment is that the logarithmic correction also enters the correlation length along the channel. We also underline that the difference between $\operatorname{Pr}<1$ an $\operatorname{Pr}>1$, so clear in the RB problem, should not matter here ( $\nu$ appears only in $L$ ).
To conclude, the agreement between the present experimental results and simple ideas on inertial convection can be obtained only if we consider that $L=\theta / 2 \beta$ is the natural coherence length controlling the convection. In our experiment, it indeed appears as a coherence time. The existence of this self-constructed vertical length $L$, different from the imposed horizontal one $d$, is an important result of this experiment, with eventual consequences for other examples of free homogeneous inertial convection. The linear relation which appears between $\mathrm{Nu}$ and $\mathrm{Re}_{n} \operatorname{Pr}$ suggests that heat fluxes can be estimated in natural situations through the relatively simple measurement of velocities and correlation length.

We are indebted to J. L. Le Mouel and F. Perrier, on one side, and to F. Toschi and D. Lohse, on the other side, for their visits and numerous enlightening discussions. Thanks are also due to F. Dumas, F. Vittoz, and M. Moulin for technical assistance. This work was supported by the Région Rhône Alpes, under Emergence Contract No. 301491302.

*Electronic address: Mathieu.Gibert@ens-lyon.fr

[1] E. Siggia, Annu. Rev. Fluid Mech. 26, 137 (1994).

[2] X. Chavanne, F. Chillà, B. Chabaud, B. Castaing, and B. Hébral, Phys. Fluids 13, 1300 (2001).

[3] E. Calzavarini, D. Lohse, F. Toschi, and R. Tripiccione, Phys. Fluids 17, 055107 (2005).

[4] F. Perrier, P. Morat, and J. L. Le Mouel, Phys. Rev. Lett. 89, 134501 (2002).

[5] D. Lohse and F. Toschi, Phys. Rev. Lett. 90, 034502 (2003).

[6] M. Debacq, V. Fanguet, J. P. Hulin, D. Salin, and B. Perrin, Phys. Fluids 13, 3097 (2001).

[7] M. Debacq, J. P. Hulin, D. Salin, B. Perrin, and E. J. Hinch, Phys. Fluids 15, 3846 (2003).

[8] J.H. Arakeri, F.E. Avila, J. M. Dada, and R. O. Tovar, http://eprints.iisc.ernet.in/archive/00001685/01/ convection.pdf.

[9] Typical values are $2 \mathrm{k} \Omega$ at $20^{\circ} \mathrm{C}$ and $1 \mathrm{k} \Omega$ at $40^{\circ} \mathrm{C}$.

[10] MATLAB toolbox.

[11] LaVision DaVis 7, C2004 LaVision GmbH. 